\title{
Design Improvements of Acoustic Response of an Automotive Air Intake System
}

\author{
Jin Teck Yap ${ }^{1}$, Noor Zafirah Abu Bakar ${ }^{1 *}$, Nur Fadzilah Shaikh Dawood ${ }^{2}$ and Muhammad \\ Adam Rosli ${ }^{2}$ \\ ${ }^{1}$ Scool of Computer Science and Engineering, Taylor's University Lakeside Campus, No. 1 Jalan \\ Taylor's 47500 Subang Jaya, Selangor, Darul Ehsan, Malaysia. \\ ${ }^{2}$ Proton Holdings, Hicom Industrial Estate, 47600 Shah Alam, Selangor, Malaysia.
}

\begin{abstract}
This study aims to improve the acoustic response of an automotive air intake system (AIS) using harmonics acoustics analysis system. Noise pollution imposes adverse health effects on human beings. One of the sources of noise pollution comes from the transportation sector. Combustion engines produce noise through the act of combustion during operation. Noise generated by the AIS affects the comfort and long-term health of humans during operations. The noise produced by the AIS can be reduced through the introduction to resonators. A baseline result was modelled through creating an internal cavity and running a harmonics acoustic analysis simulation in ANSYS. Transmission loss and frequency response data was analysed as well as the location of the noise through visual contour. Modifications to the baseline geometry were done through the addition of a resonator targeted at $197 \mathrm{~Hz}$ and the geometry was then analysed again through the same parameters with harmonics acoustics analysis. The results show the resonator is effective with the peak in noise at $197 \mathrm{~Hz}$ reduced but additional noise is introduced as a result of the addition of a resonator into the AIS.
\end{abstract}

\section{Introduction}

Noise pollution has been shown to have adverse effects on the health of human being [1]. The noise humans are exposed to everyday especially in the city can lead to a wide range of health effects such as sleep disturbance, tinnitus, cognitive impairment in children and annoyance. It is important to reduce the noise pollution in everyday lives to improve the health of humans. Regulations are set by the government [2] to ensure that noise levels in cities can be controlled. There are many sources of environmental noise that can lead to adverse health effects. One of the sources is from the transportation sector. Current motor vehicles are powered by combustion engines. Combustion engines produce noise from various parts such as the engine, exhaust system, and the air intake system (AIS). An AIS generates noise through the resonance produced by the engine during operation.

The AIS is responsible for drawing ambient air and directing it into the engine, allowing for smooth and consistent airflow that can be used to create power through the combustion

Corresponding author: noorzafirah.abubakar@taylors.edu.my 
of an internal-combustion engine (ICE). An AIS consists of a snorkel, intake tube, air filter, airbox and zip tube as seen in Figure 1.

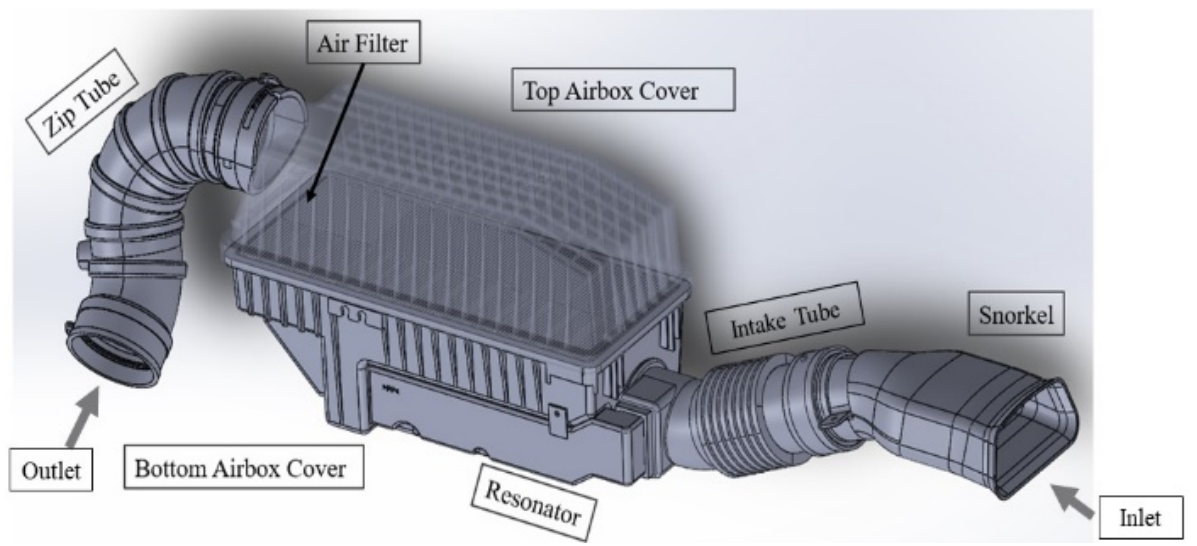

Fig. 1. Model of AIS of Proton Iriz 1.3 [3]

The noise of an AIS is usually measured by the sound pressure level (SPL) inside the AIS. Reduction in the SPL in an AIS can reduce the noise generated during the operation of a motor vehicle. One of the ways in reducing the SPL of an AIS is the introduction of resonators. There are two types of resonators used to improve the acoustic performance of an AIS, quarter lambda resonators and Helmholtz resonators as shown in Figure 2.

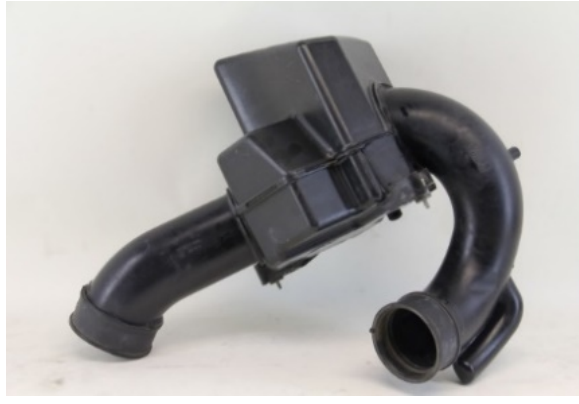

(a)

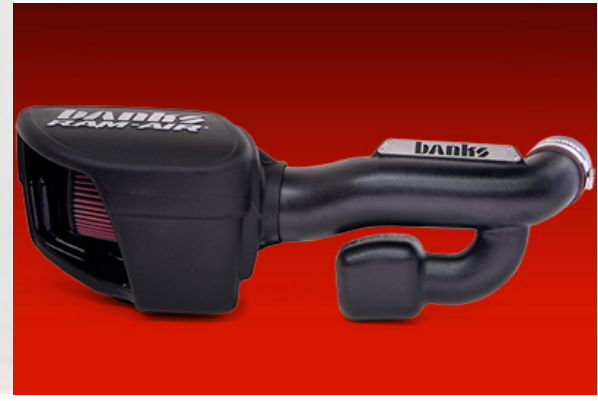

(b)

Fig. 2. Images of (a) quarter lambda resonator and (b) Helmholtz resonator

Resonators besides affecting the acoustic performance of the AIS, also affect the volumetric efficiency of the AIS [4]. A reduce in volumetric efficiency may affect the output of the powertrain, forcing the driver to the vehicle to put more load into the powertrain, raising the noise of the powertrain. It is possible to minimize the efficiency loss of the AIS through various configurations of the resonators.

In a conventional ICE, four stroke cycles are sued to create power, these four strokes are intake, compression, combustion, and exhaust stokes. During the intake stroke, the intake valves open to allow for air and fuel mixture to enter the combustion chamber from the intake manifold. As the intake valves close during the compression stroke, pressure builds up in the intake manifold due to the closed valves. The increase in pressure forces air to travel out of the intake manifold, back to the snorkel of the air intake, creating noise. This is visualized in Figure 3 . 

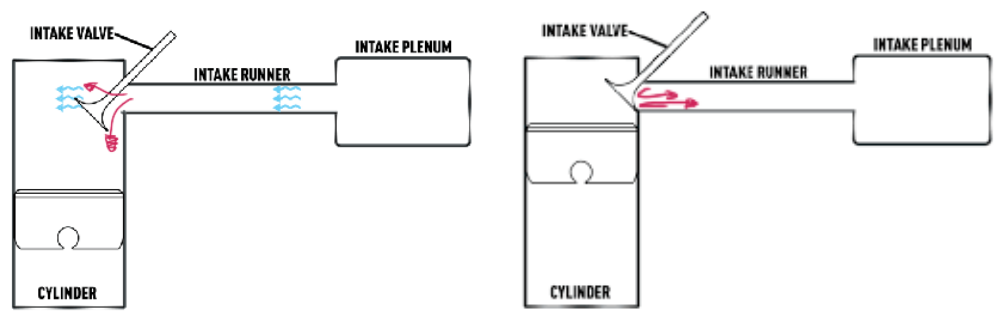

Fig. 3. Image showing the pressure build up when valve closes during compression [5]

\subsection{Research objectives}

The objectives of this research are:

1. To develop a validated acoustic simulation model of an AIS to identify the location of the highest noise along an AIS.

2. To modify the current AIS by adding resonators at the identified areas to improve acoustic performance.

\section{Research Methodology}

As this project is entirely software-based, the flowchart Figure 4 shows the process and setup that were followed to complete the research. The geometry for the baseline air intake was created in SolidWorks. Then the geometry exported into ANSYS [6] for meshing and Harmonics Acoustic analysis module was used in this research to analyse the acoustic response of the AIS and compared with measured data to understand the accuracy of the simulation.

SolidWorks 2018 [7] was used to create the design improvements based on the baseline design. The improved designed underwent the same analysis as the baseline model in ANSYS to obtain results for comparison.

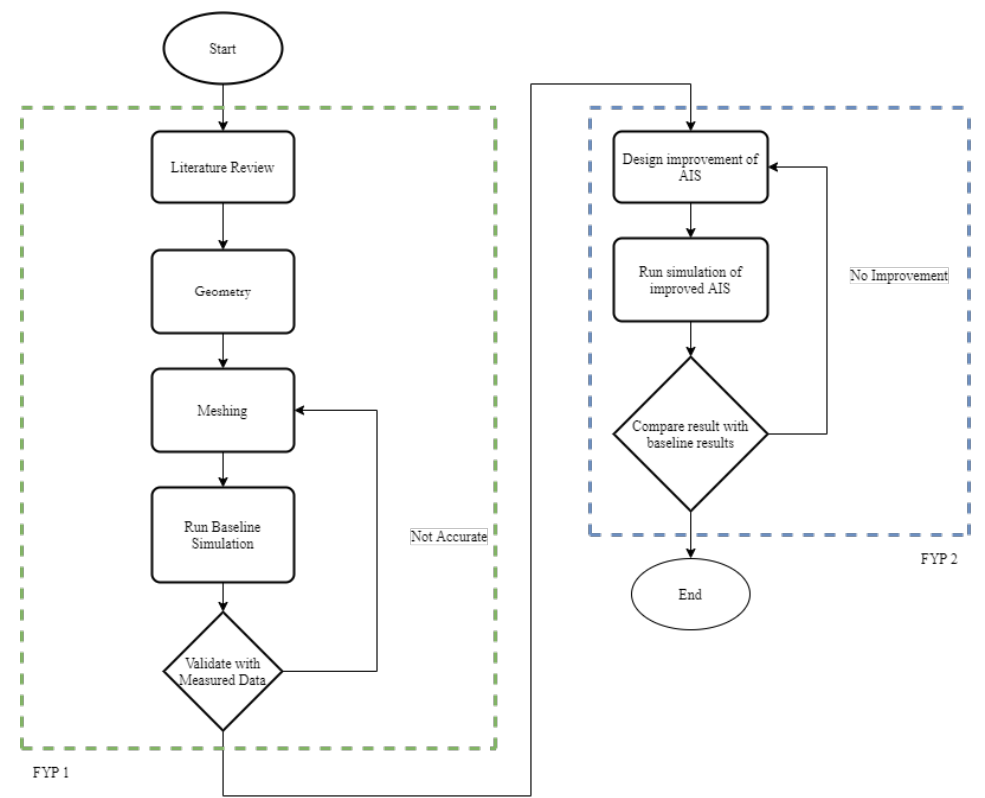

Fig. 4. Flow chart of research 


\subsection{Obtaining baseline results}

The simulation was conducted using Harmonics Acoustic analysis system in ANSYS. An internal cavity was created from the geometry of the model designed. The mesh sizing was set at $0.02 \mathrm{~mm}$ for the baseline simulation to have a balance of accuracy and computing time.

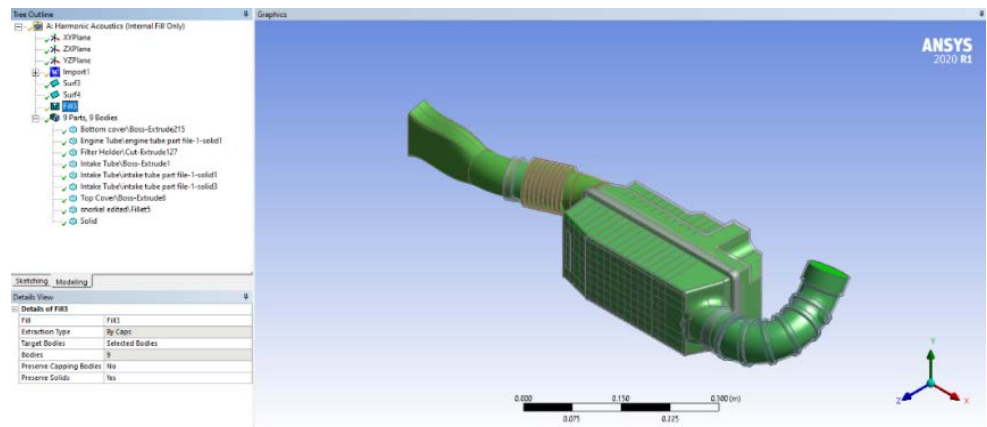

Fig. 5. Creation of Internal Cavity of AIS in ANSYS

The parameters set for the simulation were as follows:

- Material of internal cavity: Air

- Temperature of internal cavity: $20^{\circ} \mathrm{C}$

- Acoustic source: $343.15 \mathrm{~ms}^{-1}$ surface velocity at zip tube.

The geometry material was determined to be air as it represented the internal cavity of the AIS. The surface velocity of the sound source was set at the speed of sound when the temperature was at $20^{\circ} \mathrm{C}$. The frequency range of the analysis was set at a range of $0 \mathrm{~Hz}$ to $500 \mathrm{~Hz}$, and an interval of $0.5 \mathrm{~Hz}$ was determined for the simulation to have a more accurate reading of the acoustic performance of the AIS.

The transmission loss of the AIS and the frequency response of the AIS were gathered in the simulation to understand the acoustic performance of the AIS. Transmission loss was measured by the difference in sound levels between two points [4]. A transmission loss graph shows the ability of the medium in absorbing sound at a specific frequency. A peak in a transmission loss graph shows a strong absorption of sound while a dip shows an inefficiency of sound absorption. To obtain transmission loss results in ANSYS, ports must be defined in the analysis module. A port surface was selected at the surface of the snorkel while another port surface was selected at the surface of the zip tube. Both ports shared the same surface body. The setup can be seen in Figure 6. When setting up the results for transmission loss, the inlet was defined at the zip tube while the outlet was defined at the snorkel as seen in Figure 7.

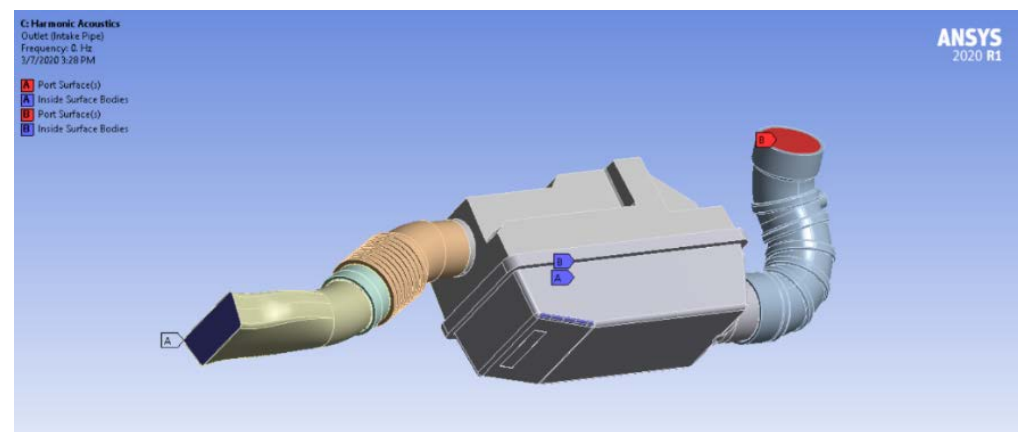

Fig. 6. Placement of ports on AIS 


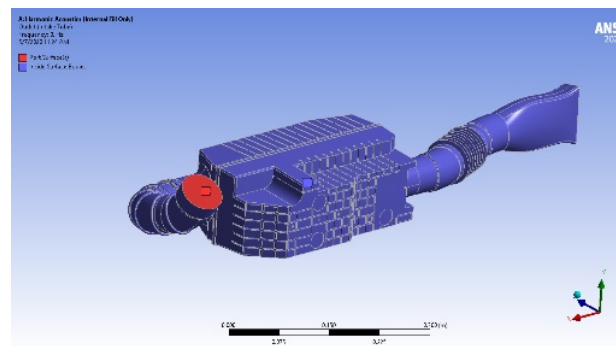

(a)

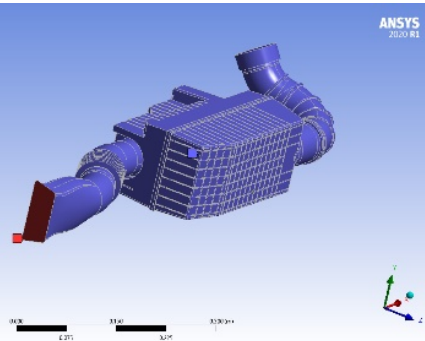

(b)

Fig. 7. Configuration of (a) input and (b) output for transmission loss

The frequency response of the AIS can be captured on any desired surface of the geometry. The frequency response of the AIS was captured at the snorkel of the AIS to determine the acoustic response of the AIS perceived by the environment as the zip tube leads to the inlet of the engine. After determining the peaks in the sound pressure levels inside the AIS, contour images of the frequency response of the AIS at peaks in the sound pressure levels were evaluated to understand the areas where the AIS shows to have high resonance in a specific frequency as show in Figure 8.

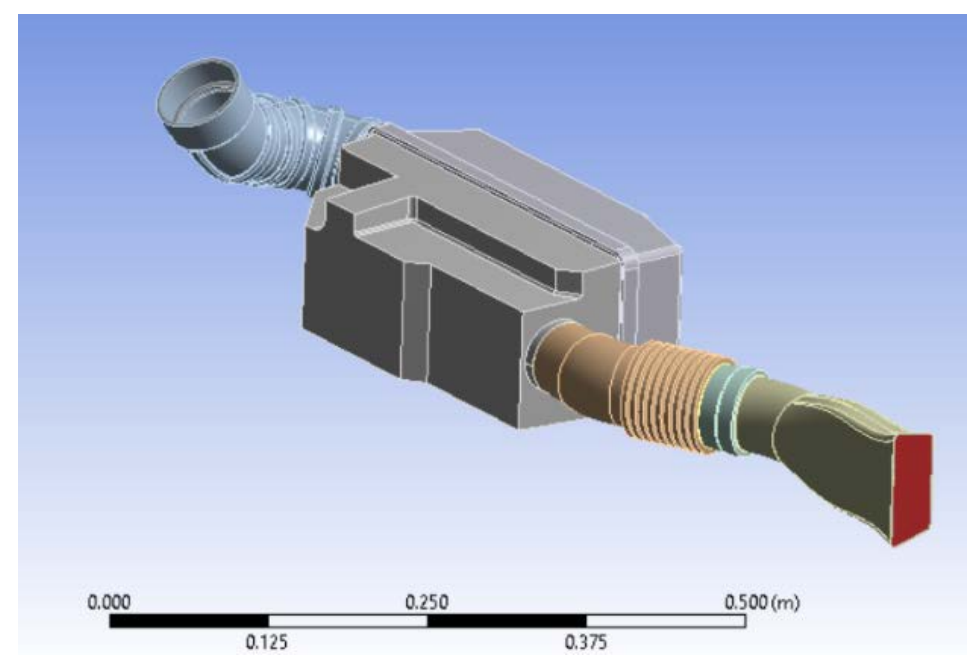

Fig. 8. Frequency Response Measured at Surface of Snorkel

\section{Results}

\subsection{Baseline results}

The transmission loss and frequency response of the baseline geometry were recorded and plotted into separate graphs as seen in Figure 9 and Figure 10. The results show that there are two points between $0 \mathrm{~Hz}$ to $500 \mathrm{~Hz}$ range. These points were determined to be $197 \mathrm{~Hz}$ and $304 \mathrm{~Hz}$. 


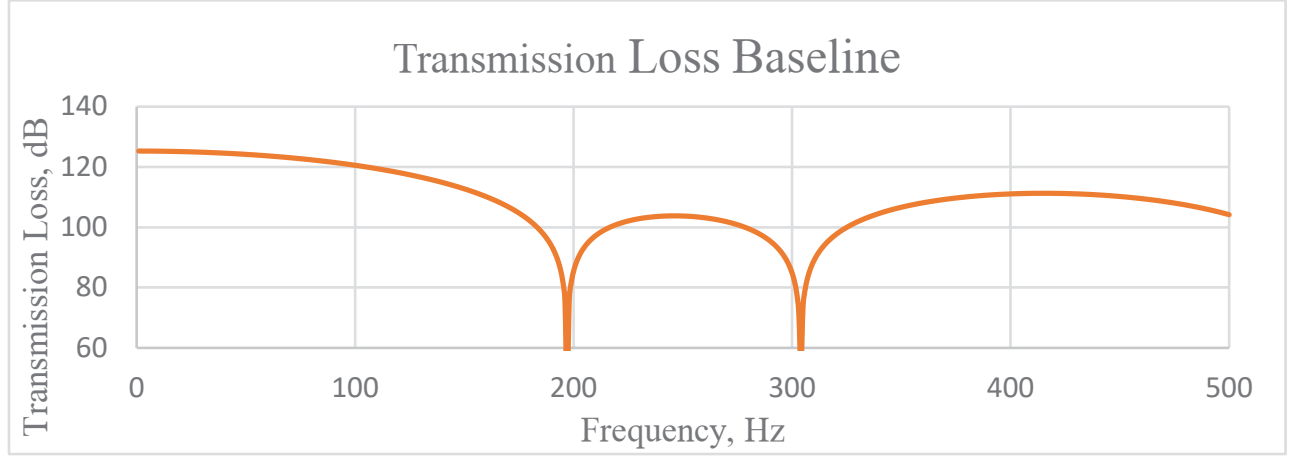

Fig. 9. Graph showing the transmission loss of baseline geometry

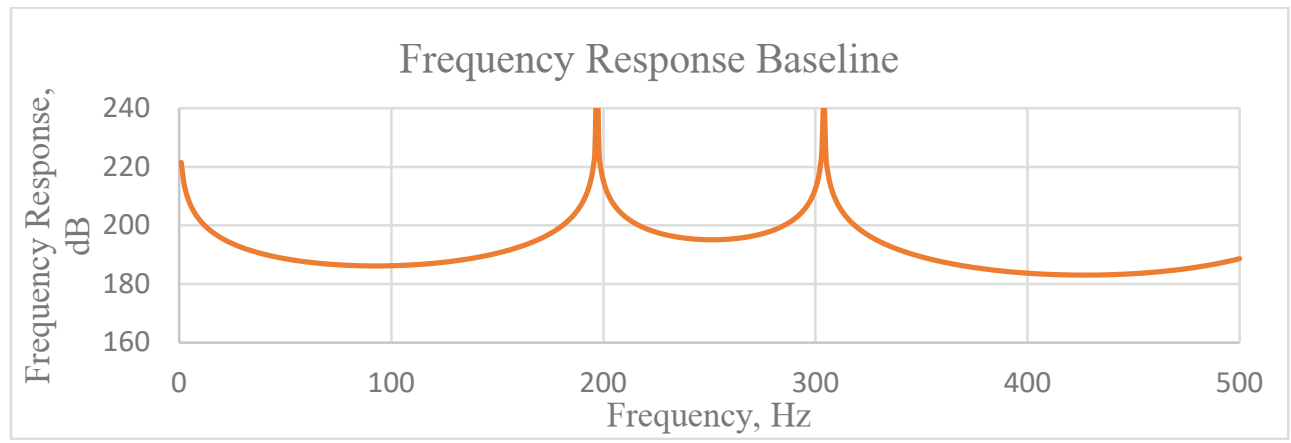

Fig. 10. Graph showing the frequency response of baseline geometry

To locate the location along the AIS where the resonance was present, a visual contour was created. The visual contours generated from the data set at $197 \mathrm{~Hz}$ and $304 \mathrm{~Hz}$. The visual contour is visualized across the whole AIS as shown in Figure 11.

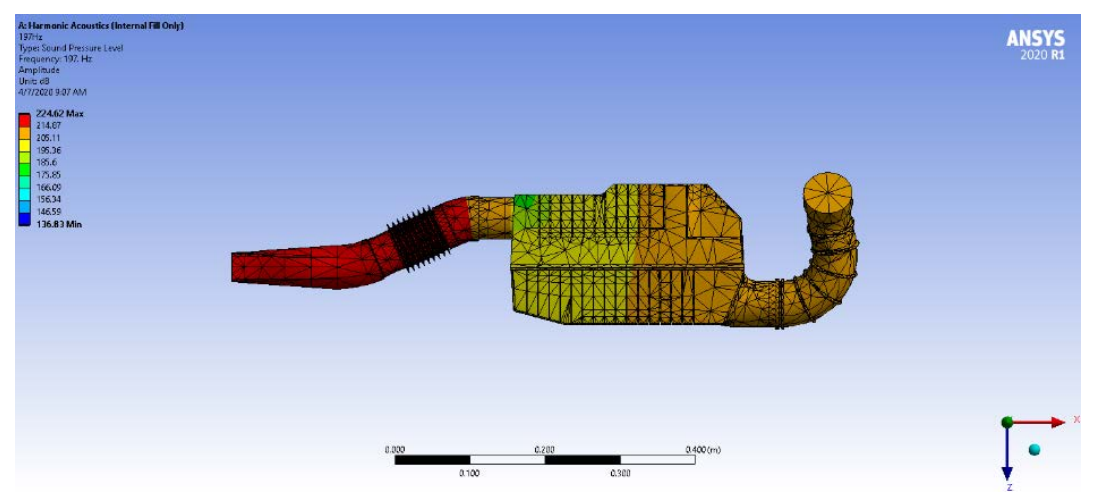

Fig. 11. Visual contour frequency response at $197 \mathrm{~Hz}$

The visual contour shows at $197 \mathrm{~Hz}$, the region near the snorkel show to have high resonance. To tackle the resonance at this frequency, a resonator was designed and placed along this area.

\subsection{Modifications to the baseline geometry}

The equation to calculate the effective frequency of a Helmholtz resonator is as below [8]: 


$$
\mathrm{f}=\frac{\mathrm{c}}{2 \pi} \sqrt{\frac{S}{V L}}
$$

Where

$f=$ Effective frequency of resonator, $\mathrm{Hz}$

$c=$ speed of sound, ms-1

$S=$ Area of neck, $\mathrm{m} 2$

$V=$ Volume of resonator, $\mathrm{m} 3$

$L=$ length of neck, $\mathrm{m}$

The speed of sound, $c$, is set at $343 \mathrm{~m} / \mathrm{s}$ as the temperature of the AIS was set at $20^{\circ} \mathrm{C}$.

When targeting the frequency of $197 \mathrm{~Hz}$, the equation can be simplified to:

$$
\frac{S}{V L}=13.023
$$

The area of the neck was constrained by the size of the intake tube. The diameter of the neck is set at $0.015 \mathrm{~m}$. The area of the neck was calculated to be $1.767 \times 10-4 \mathrm{~m}^{2}$.

$$
\begin{gathered}
d_{\text {neck }}=0.015 \mathrm{~m} \\
S=\frac{\pi d^{2}}{4} \\
S=1.767 \times 10^{-4} \mathrm{~m}^{2}
\end{gathered}
$$

To reduce the volume of the resonator, the length of the neck was increased. The length of the next was set at $0.1235 \mathrm{~m}$ and the radius of the resonator was set at $0.03 \mathrm{~m}$.

$$
\begin{gathered}
L=0.1235 \mathrm{~m}^{2} h \\
V=\pi r_{\text {resonator }}{ }^{2} \\
\frac{1.767 \times 10^{-4} \mathrm{~m}^{2}}{\pi 0.03^{2} h(0.1235)}=13.023
\end{gathered}
$$

With that, the height of the resonator was determined to be $38.86 \mathrm{~mm}$. The parameters of the resonator were then implemented into the intake tube near the snorkel using SolidWorks as shown in Figure 12.

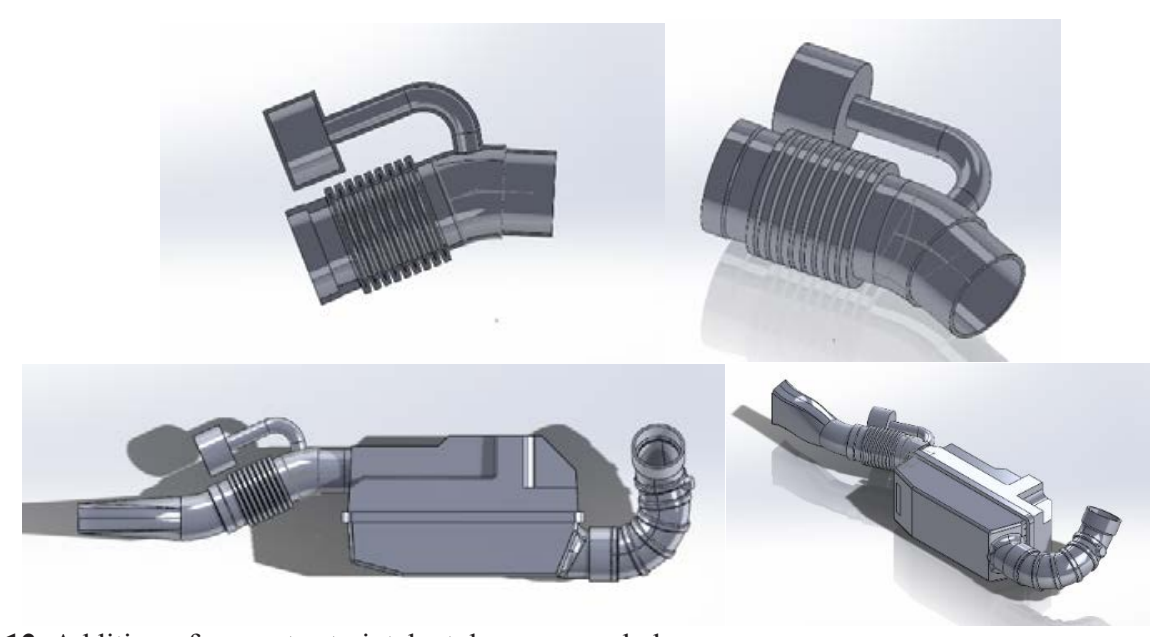

Fig. 12. Addition of resonator to intake tube near snorkel 
Similar to the baseline geometry, an internal cavity was created from the geometry and the mesh was set at a body sizing of $0.02 \mathrm{~mm}$. The parameters set for this simulation were similar to the parameters set for the baseline simulation. This is done to ensure consistency and accuracy of the results when comparing both simulations.

\section{Results and Discussions}

Transmission loss and acoustic response were gathered in this analysis and separated graphs were plotted in Figure 13 and Figure 14. Looking at the results gathered from the analysis, the resonator was able to suppress the noise at $197 \mathrm{~Hz}$ effectively. This can be seen by the suppressed peak in sound pressure levels that $197 \mathrm{~Hz}$. The visual contour of the AIS as seen in Figure 15 also show that there is a significant difference when compared to the original analysis. The air duct shows a significant lower sound pressure level compared to the baseline AIS. There is a $20 \mathrm{~dB}$ difference in the noise output at $197 \mathrm{~Hz}$ after the implementation of the resonator as shown in Figure 16 and Figure 17.

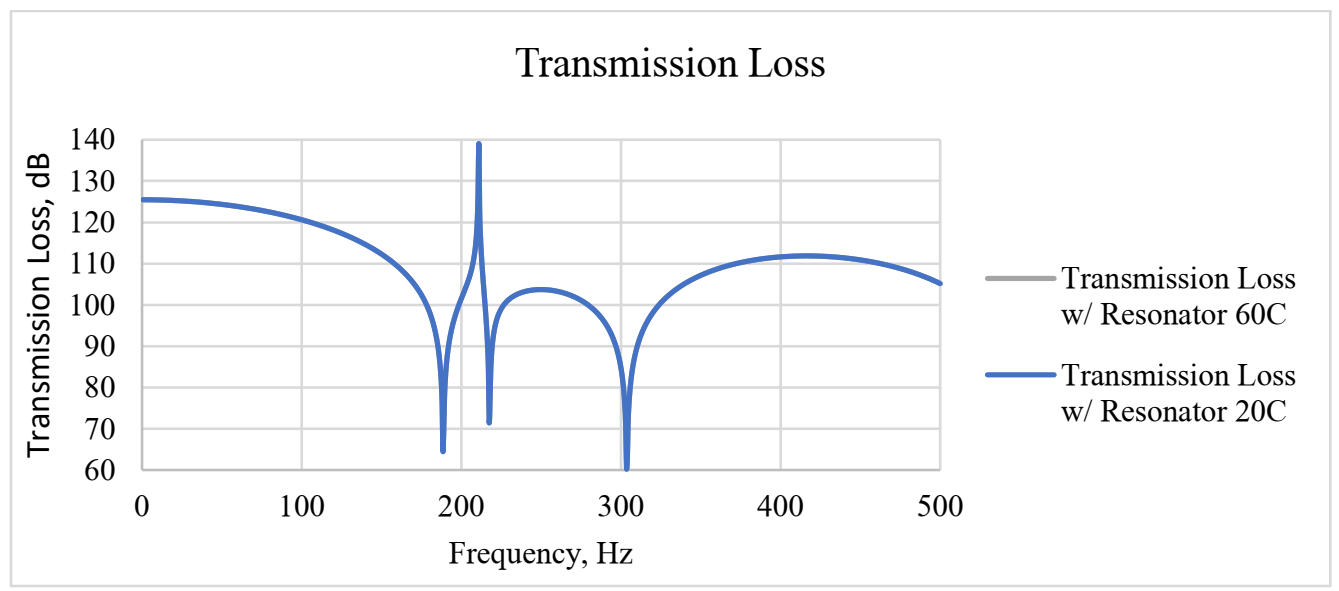

Fig. 13. Transmission loss of AIS after the implementation of a resonator

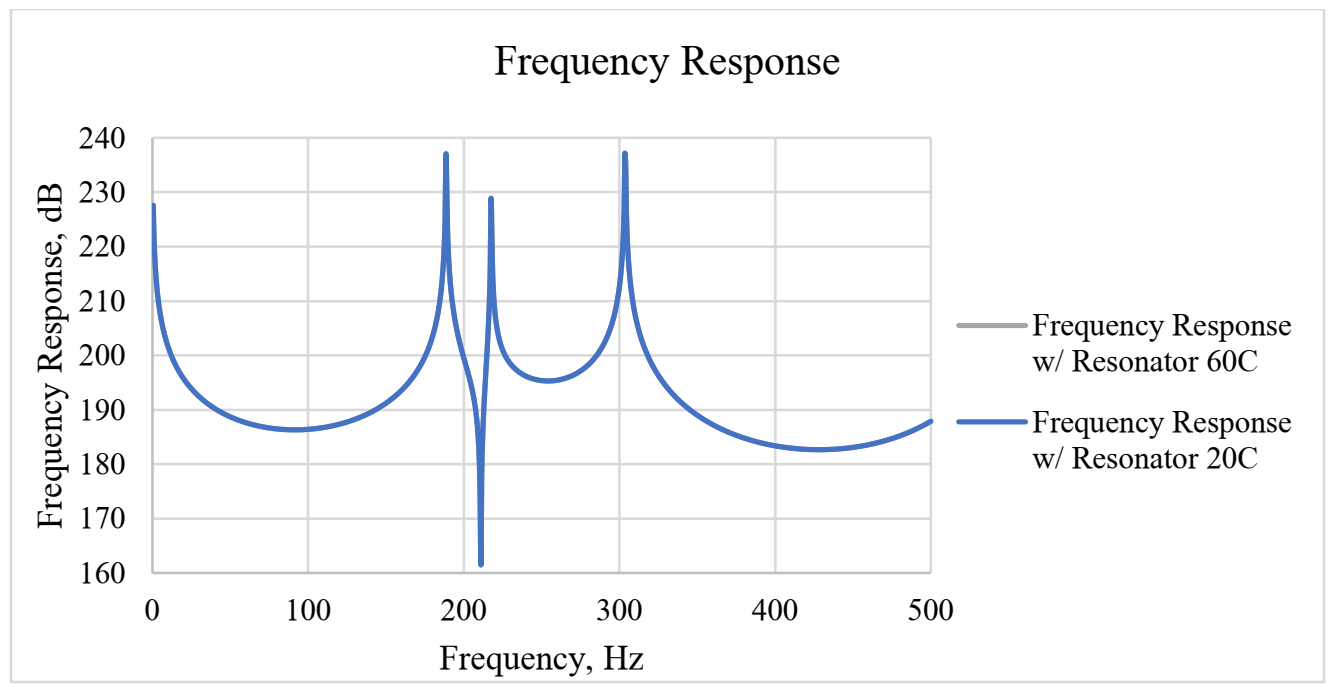

Fig. 14. Frequency Response of AIS after the implementation of a resonator 


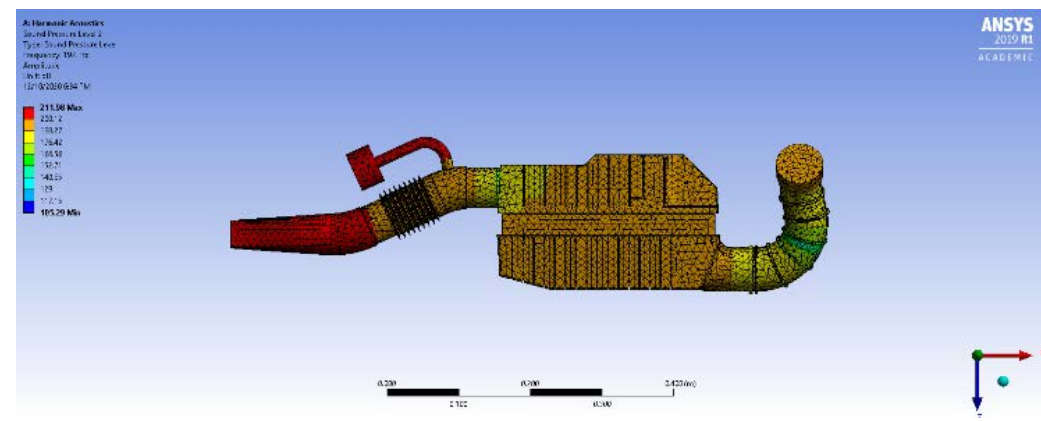

(a)

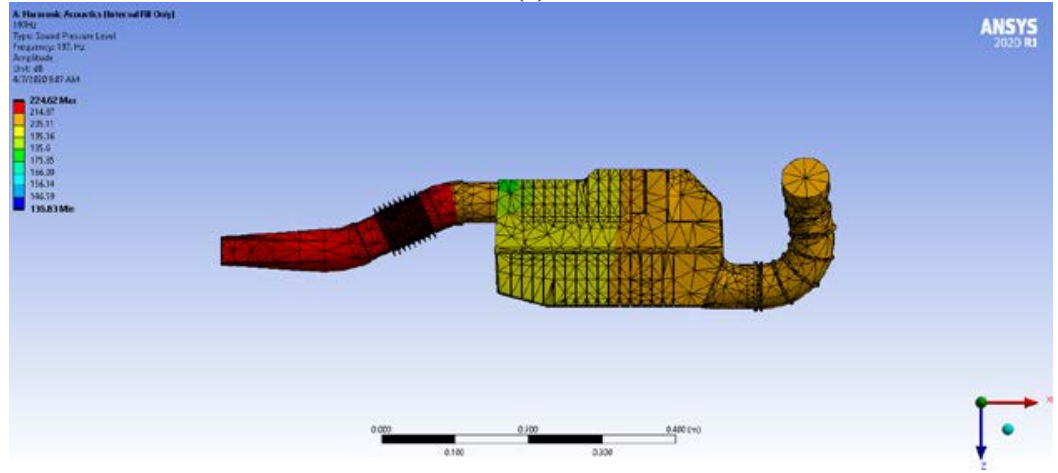

(b)

Fig. 15. Comparison of (a) AIS with resonator and (b) original AIS

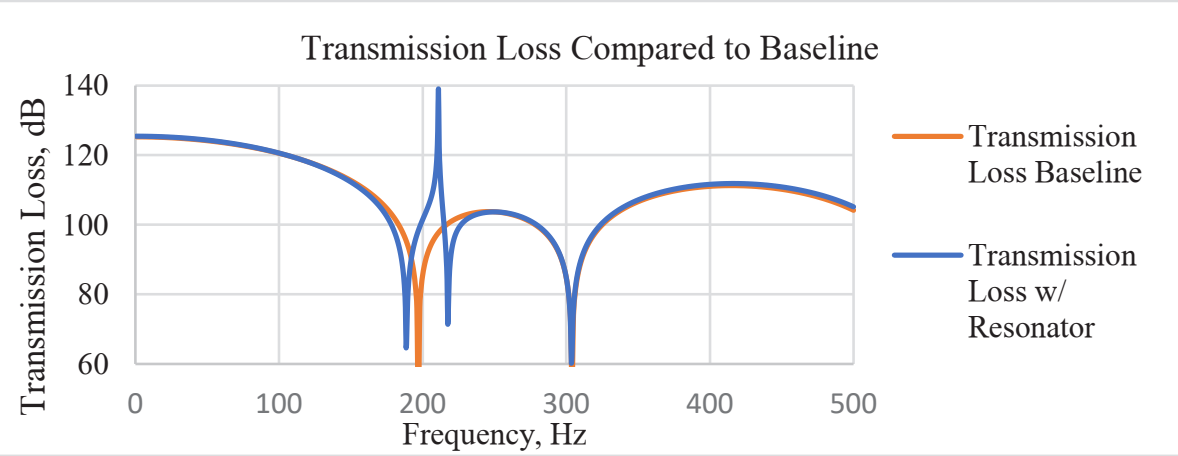

Fig. 16. Transmission loss of modified AIS when compared to baseline

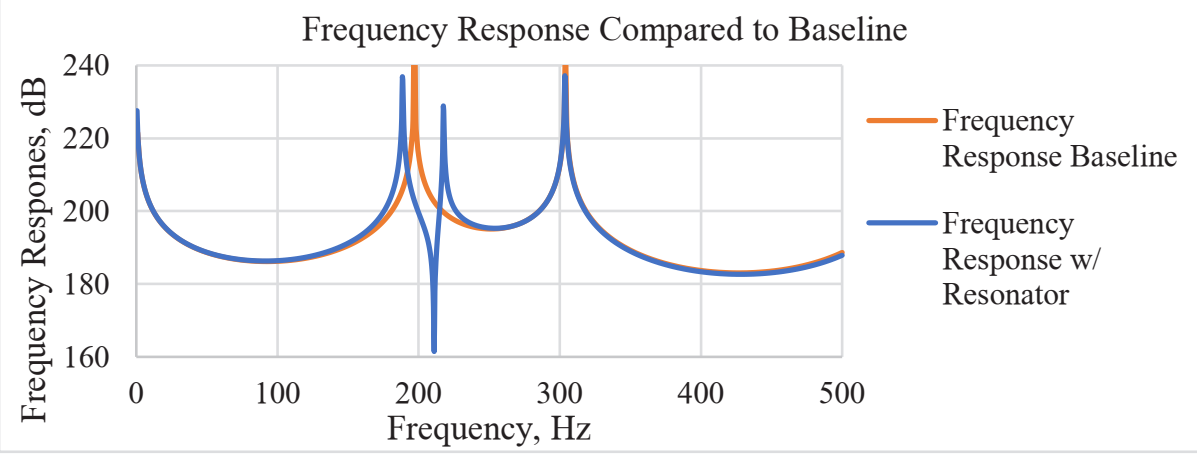

Fig. 17. Frequency response of modified AIS when compared to baseline 
However, two additional peaks have emerged at frequencies $188 \mathrm{~Hz}$ and $217 \mathrm{~Hz}$. Although the peak noise of both frequencies are lower than the peak noise at $197 \mathrm{~Hz}$, the addition of two frequencies may affect the perceived loudness of the AIS. When comparing the results of this analysis with the research done in [9], the characteristics of the Helmholtz resonator can be seen present at the research done. The results of the research show a similar pattern that can be seen when a Helmholtz resonator is introduced into the AIS to tackle resonance. This result shows that the simulation model is accurate in replicating the effects of a Helmholtz resonator as the introduction of the Helmholtz resonator is able to tackle the resonance at $197 \mathrm{~Hz}$. The effects of the introduction of the Helmholtz resonator can be seen in Figure 18.

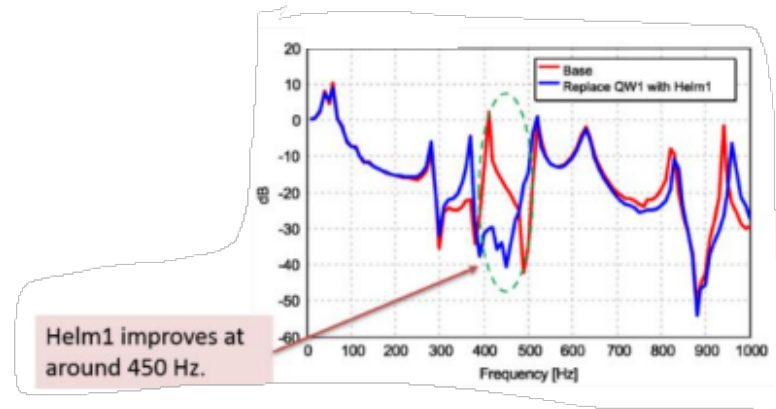

Fig. 18. Effects of replacing QW1 with Helmholtz resonator [9]

\section{Future Work}

To improve the acoustic performance of the AIS further, adjustments to the geometry of the Helmholtz resonator can be made to reduce the additional noise when implementing a Helmholtz resonator. The use of other types of resonators such as a half-wavelength resonator or a quarter-wavelength resonator can also be explored to understand the effects of other types of resonators to the acoustic performance of the AIS.

\section{References}

1. F. Lin, World Health Organization, Regional Office for Europe (2011)

2. Guidelines for Environmental Noise Limits and Control, 3rd ed. Putrajaya, Malaysia: Department of Environment Malaysia (2019)

3. G. Ronald Han-Bo, Thesis, Taylor's University (2019)

4. K. Mohd Nasir, I. Moumen, A. Muhammad Iqbal, and R. Zairi Ismael, ARPN J. Eng. Appl. Sci, 10, 20 (2015)

5. S. Bryson, Mishimoto Engineering Blog (2019)

6. ANSYS Manual (2020)

7. SOLIDWORKS Manual (2020)

8. M.L. Munja, Acoustics of Ducts and Mufflers (1987)

9. A. A. Dahlan, M. F. Muhamad Said, Z. A. Latiff, M. R. Mohd Perang, S. A. Abu Bakar, and R. I. Abdul Jalal, Int. J. Automot. Mech. Eng, 16, 1 (2019) 